\title{
Advanced electron cyclotron heating and current drive experiments on the stellarator Wendelstein $7-X$
}

\author{
Torsten Stange ${ }^{1, *}$, Heinrich Peter Laqua ${ }^{1}$, Marc Beurskens ${ }^{1}$, Hans-Stephan Bosch $^{1}$, Sergey Bozhenkov ${ }^{1}$, Rudolf $_{\text {Brakel }}{ }^{1}$, \\ Harald Braune ${ }^{1}$, Kai Jakob Brunner ${ }^{1}$, Alvaro Cappa ${ }^{2}$, Andreas Dinklage ${ }^{1}$, Volker Erckmann ${ }^{1}$, Golo Fuchert ${ }^{1}$, \\ Gerd Gantenbein ${ }^{4}$, Florian Gellert ${ }^{1}$, Olaf Grulke ${ }^{1}$, Dirk Hartmann ${ }^{1}$, Matthias Hirsch $^{1}$, Udo Höfel ${ }^{1}$, Walter Kasparek ${ }^{3}$, \\ Jens Knauer ${ }^{1}$, Andreas Langenberg ${ }^{1}$, Stefan Marsen ${ }^{1}$, Nikolai Marushchenko ${ }^{1}$, Dmitry Moseev ${ }^{1}$, Novomir Pablant ${ }^{5}$, \\ Ekkehard Pasch $^{1}$, Kian Rahbarnia ${ }^{1}$, Humberto Trimino Mora ${ }^{1}$, Toru Tsujimura ${ }^{6}$, Yuriy Turkin ${ }^{1}$, Tom Wauters ${ }^{7}$, \\ Robert $\mathrm{Wolf}^{1}$, \& the W7-X-Team

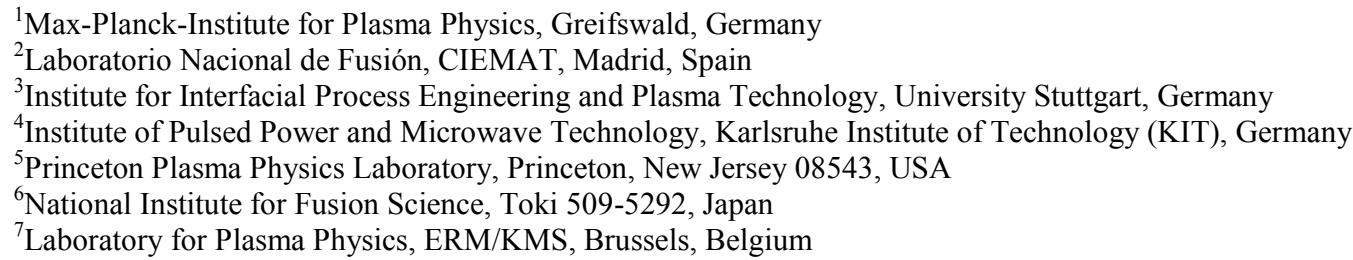

\begin{abstract}
During the first operational phase (OP 1.1) of Wendelstein 7-X (W7-X) electron cyclotron resonance heating (ECRH) was the exclusive heating method and provided plasma start-up, wall conditioning, heating and current drive. Six gyrotrons were commissioned for OP1.1 and used in parallel for plasma operation with a power of up to 4.3 MW. During standard X2-heating the spatially localized power deposition with high power density allowed controlling the radial profiles of the electron temperature and the rotational transform. Even though W7-X was not fully equipped with first wall tiles and operated with a graphite limiter instead of a divertor, electron densities of $n_{e}>3 \cdot 10^{19} \mathrm{~m}^{-3}$ could be achieved at electron temperatures of several $\mathrm{keV}$ and ion temperatures above $2 \mathrm{keV}$. These plasma parameters allowed the first demonstration of a multipath $\mathrm{O} 2$-heating scenario, which is envisaged for safe operation near the X-cutoff-density of $1.2 \cdot 10^{20} \mathrm{~m}^{-3}$ after full commissioning of the ECRH system in the next operation phase OP1.2.
\end{abstract}

\section{INTRODUCTION}

On 10th December 2015 the first plasma was created in Wendelstein 7-X (W7-X). It is the world's largest optimized stellarator $(\mathrm{R}=5.5 \mathrm{~m}, \mathrm{a}=0.5 \mathrm{~m})$ with $3-\mathrm{D}$ shaped superconducting modular coils and a five-fold symmetry. W7-X aims to achieve reactor relevant plasma parameters in quasi steady state operation [1]. For this reason, the device will be equipped with a 10 MW electron cyclotron resonance heating (ECRH) system allowing at least 30 minutes continuous operation. The design frequency of the 10 gyrotrons already installed is $140 \mathrm{GHz}$ corresponding to second harmonic heating at the magnetic axis with a local magnetic field of $2.5 \mathrm{~T}$ [2]. As a very versatile technique, ECRH can provide plasma start-up, bulk plasma heating, current drive as well as wall conditioning. Even though, the confinement of a stellarator is only given by the external field coils, electron cyclotron current drive $(\mathrm{ECCD})$ can be used at W7-X to compensate a finite bootstrap current of up to tens of kilo-Ampéres in some magnetic field configurations, which can cause a shift of the strike line on the divertor. Furthermore, the steady state operating magnetic field prevents any re-conditioning of the wall by glow discharges, requiring the development of adapted ECRH wall conditioning scenarios.

ECRH was the exclusive heating system in the first operational phase (OP1.1) which ended in March 2016 after 10 weeks of operation and was primarily dedicated to an integral commissioning of the heating system, the diagnostics and especially the components of the device itself, e.g. vacuum vessel and coil system. The machine was not fully equipped with wall protecting graphite tiles leaving a larger surface of the $\mathrm{CuCrZr}$-cooling structure unprotected. Furthermore, instead of the foreseen 10 divertor elements, a graphite limiter was installed on the high field side (HFS) in each bean plane of the five modules preventing a direct contact of the plasma with the unprotected regions of the plasma vessel [3]. Therefore, the plasma volume was limited to an effective minor radius of $\mathrm{a}=0.49 \mathrm{~m}$. In parallel, the flexible coil system of W7-X was used to operate a magnetic field configuration at a lower rotational transform developing 


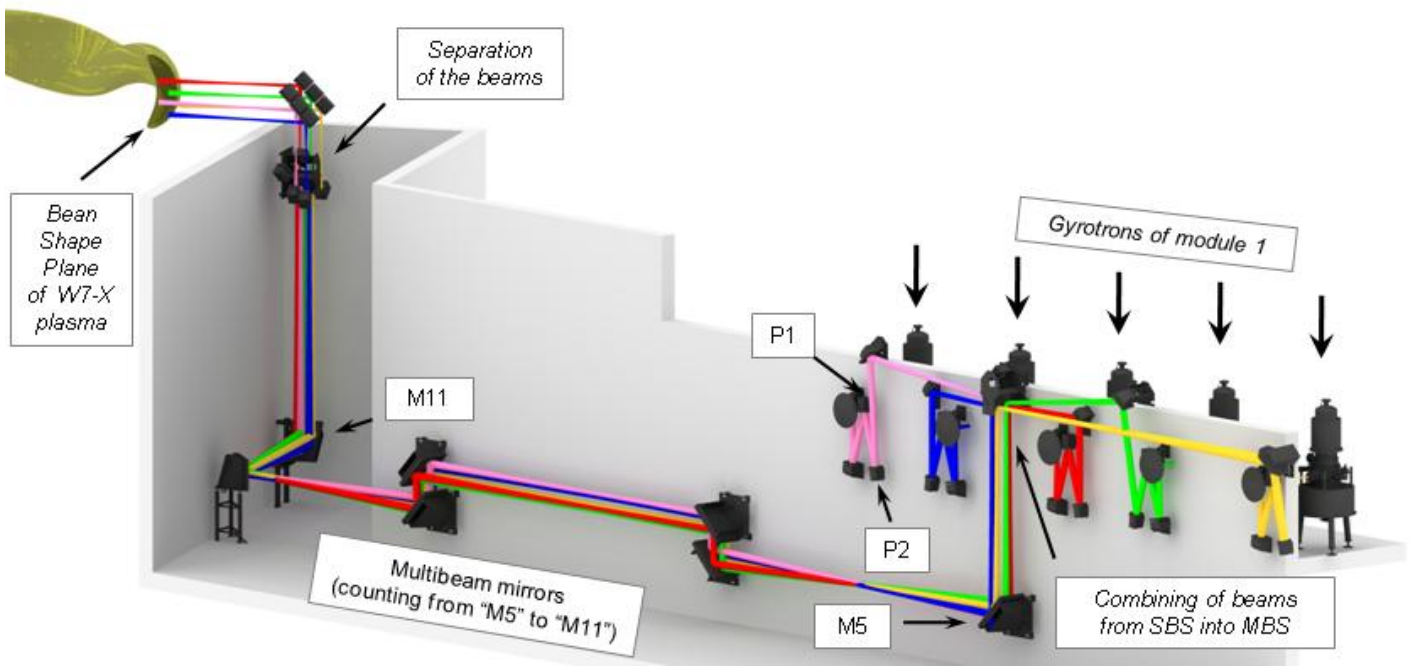

Fig. 1. Fully quasi-optical transmission line to the torus of Wendelstein 7-X

from $t_{0}=0.79$ in the center to $t_{a}=0.87$ at the scrape off layer to push the $5 / 6$ island chain inside the plasma volume.

\section{Setup of the ECRH system}

The heating beams are provided by 10 gyrotrons, which are aligned in two symmetrical rows. The gyrotron outputs of each row face each other into a beam channel with two identical quasi-optical multi-beam transmission lines, one for each gyrotron row. Fig. 1 shows one half of the whole installation excluding periphery like cooling and high voltage supplies. The beam line is designed such that an additional gyrotron can be installed on each side during a later upgrade.

\subsection{Gyrotrons}

The gyrotrons were developed within the PMW-project ("Projekt Mikrowellenheizung für W7-X") at KIT and have a nominal power of $1 \mathrm{MW}$ at $140 \mathrm{GHz}$ [4]. Nine gyrotrons were built by THALES in France and another one by CPI in the USA. The overall power of all gyrotrons is up to $9 \mathrm{MW}$ in short pulse operation and up to $8 \mathrm{MW}$ for steady state. The power can be modulated with arbitrary waveforms at frequencies up to $10 \mathrm{kHz}$ to determine the power deposition zone within the plasma and conduct advanced heat wave experiments. In OP1.1 only 6 gyrotrons were in operation. This was sufficient, as the $4 \mathrm{MJ}$ per discharge given by the finite heat capacity of the limiter elements in the plasma vessel did not require more gyrotrons.

\subsection{Transmission Line}

Both quasi-optical transmission lines to the plasma vessel were part of the PMW-project, as well, and are first-of-its-kind systems. Each of them consists of five single beam sections (SBS) matching the non-perfect Gaussian beam output of the gyrotrons to the subsequent
$40 \mathrm{~m}$ long multi-beam section (MBS), which is intrinsically broadband. The whole MBS consists of 7 large-area multi-beam mirrors being able to transmit 7 gyrotron beams in parallel (5 gyrotron beams + 1 optional beam line for switching one gyrotron to another launcher +1 beam line of the spare box). Finally, the MBS separates the beams and directs them to their respective vacuum window in a single beam line. The windows interface to the four equatorial launchers installed on W7-X with three beam lines each (see Fig. 2). The plasma facing mirror of each beam line is steerable in the poloidal and toroidal direction. This way, the radial heat deposition in the plasma and the plasma current drive can be varied, respectively. The polarization of the beams can be arbitrarily adjusted by two grooved mirrors, the polarizer $1(\mathrm{P} 1)$ and polarizer 2 (P2), generating a $90^{\circ}$ - and $180^{\circ}$-phaseshift, respectively.

The beam power of each beam line is measured by a calorimetric load before entering the MBS, as depicted in Fig. 3. The measured value has to be corrected by the theoretical transmission losses up to the vessel window to determine the final plasma heating power. However, the multi-beam transmission technique gives the opportunity to introduce a retroreflector at the end of the

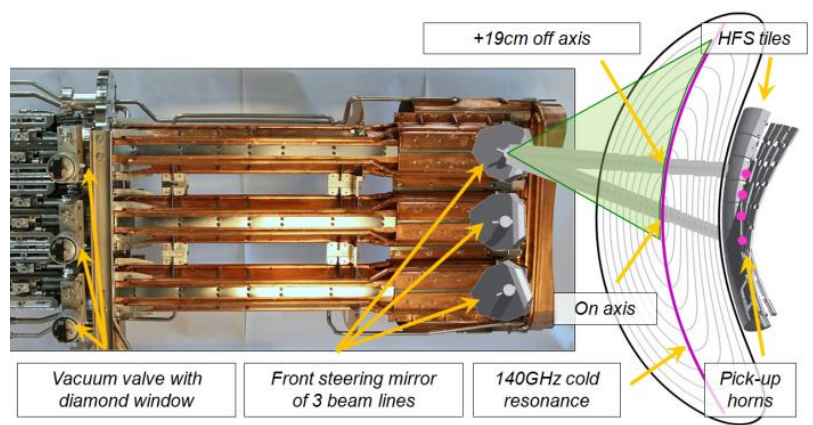

Fig. 2. Cross section of one of the 4 ECRH launchers in relation to the vacuum flux surfaces of the limiter configuration in OP1.1. The beam with a diameter of about $80 \mathrm{~mm}$ in the plasma center is shown for two examples of the upper beam line. Its whole steering range is visualized by the green shaded area. 


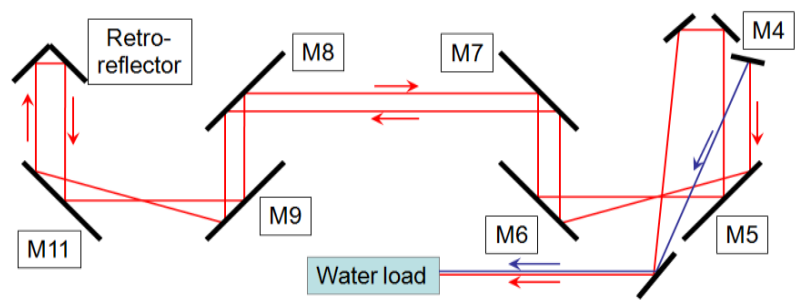

Fig. 3. Schematic picture of the MBS fed by the mirror 4 (M4) which is the last mirror of the SBS and is switchable between the water load (blue beam line) or the MBS to W7-X (red). Optional use of a retroreflector allows the additional power measurement after passing the MBS twice.

MBS to redirect a beam to the same calorimetric load for measuring the transmission losses after passing the MBS two times (see Fig. 3). Using the same load has the advantage, that uncertainties of the single power measurement cancel out. The time traces of the two separate power measurements are overlaid in Fig. 4. After 300 seconds the gyrotron as well as the load is fully equilibrated allowing an accurate comparison of both power levels. The transmission efficiency was determined for three different power levels with an averaged value of $(94.4 \pm 0.4) \%$ being in very good agreement with the theoretical value of $94.2 \%$. Therefore, the losses of the MBS can be specified to $0.06 \% / \mathrm{m}$ including atmospheric absorption of $0.02 \% / \mathrm{m}$ for $140 \mathrm{GHz}$. Finally, the overall transmission efficiency from the gyrotron window up to the launcher window can be calculated to $94 \%$ including diffraction, beam truncation, misalignment and absorption by the mirrors and by the atmosphere.

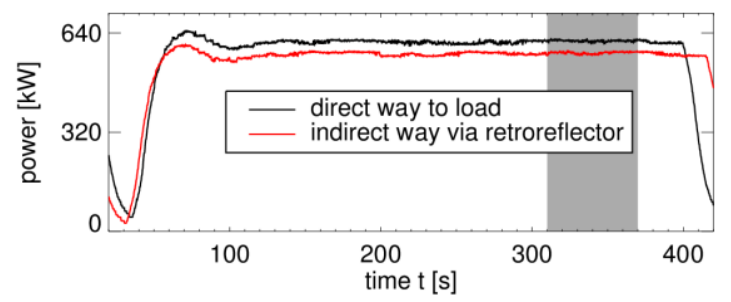

Fig. 4. Evaluation of the efficiency of the MBS by comparison of the gyrotron beam power on a direct way to the load with the indirect way via a retroflector at the end of the MBS (the evaluated time windows are shaded)



Fig. 5. Top view on the W7-X torus with the positions of the main diagnostics and the ECRH launchers in module 1 and 5.

\section{Diagnostics for plasma operation}

Fig. 5 shows an overview of the W7-X torus indicating the position of the ECRH launchers and some important diagnostics, which were commissioned during OP1.1. Safety relevant diagnostics like the stray radiation probes (sniffer) [5], indicating the ECRH absorption, and the electron cyclotron emission diagnostic (ECE) [6], measuring the electron temperature, were available from the first day of operation. A detailed description of all diagnostics is given in [7].

\section{ECRH scenarios during OP1.1}

The standard heating scenario at $\mathrm{W} 7-\mathrm{X}$ is X2-heating at $140 \mathrm{GHz}$ with an electron cutoff-density of $\mathrm{n}_{\mathrm{e}, \mathrm{X} \text {-cut }}=1.2 \cdot 10^{20} \mathrm{~m}^{-3}$. However, for electron densities above $0.8 \cdot 10^{20} \mathrm{~m}^{-3}$, which are envisaged in the future, a completely different O2-heating-scenario $\left(\mathrm{n}_{\mathrm{e}, \mathrm{O} \text {-cut }}=2.4 \cdot 10^{20} \mathrm{~m}^{-3}\right)$ should be used to prevent beam refraction or reflection in case of losing density control during the discharge. Both scenarios were successfully demonstrated up to electron densities of $\mathrm{n}_{\mathrm{e}}=4 \cdot 10^{19} \mathrm{~m}^{-3}$. The subsequent presentation follows the timeline of progress during OP1.1. The conducted heating scenarios including the expected absorption were optimized in advance with the aid of the ray tracing code TRAVIS [8] developed at IPP.

\subsection{X2-heating}

To similarly fulfil the on-axis resonance condition for thermal and suprathermal electrons during the plasma breakdown an optimal magnetic field with $\mathrm{B}_{00}=2.52 \mathrm{~T}$ on axis was forecast and proved in a magnetic field scan $(2.48-2.54 \mathrm{~T})$ on the first day of OP1.1 However, poor vacuum conditions necessitated the use of ECRH for wall conditioning during the first days of operation.

\subsubsection{Wall Conditioning}

In preparation of plasma operation, the vacuum vessel was baked one week at $150^{\circ} \mathrm{C}$ to remove most of the water. Due to possible copper sputtering and other problems with the glow discharge system, the plasma operation was started in helium without glow discharge cleaning (GDC). For this reason, the first days of plasma discharges were governed by strong outgassing of the walls, resulting in incomplete ECRH absorption and a radiative collapse immediately after breakdown. The wall conditions could be drastically improved by repeating $50 \mathrm{~ms}$ shots hundreds of times. Fig. 6 shows the full discharge behaviour after 110 pulses and in comparison the stray radiation time trace after 220 further pulses. The time period with full absorption could be doubled. All available gyrotrons were used during the cleaning pulses to bring as much energy as possible into the plasma and thus onto the plasma wetted limiter elements. Furthermore, energy is transferred to the walls by soft X-ray and charge exchange processes, thus releasing further impurities. 


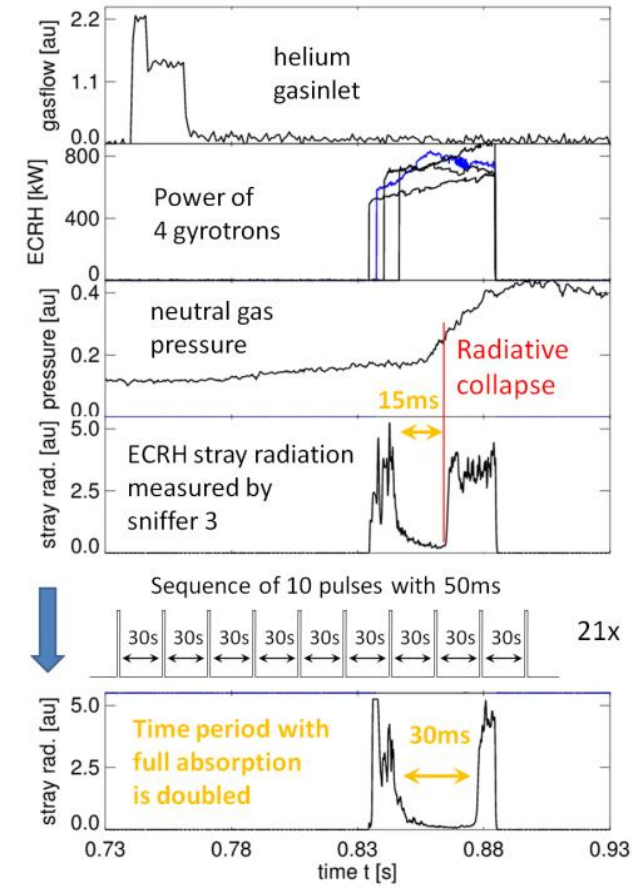

Fig. 6. Temporal evolution of a cleaning discharge @ 2.7 MW. Stray radiation after $0.1 \mathrm{~s}$ cumulated discharge time compared with $3 \mathrm{~s}$ cumulated discharge time (as published in [12])

After 3 days of W7-X operation, the glow discharge system was operated with helium for periods of $20 \mathrm{~min}$ $40 \mathrm{~min}$ in advance of each experiment day. Fig. 7 shows the value of the neutral pressure peak after a helium discharge normalized to the absorbed energy over the cumulated discharge time in which almost full absorption was achieved. After GDC the outgassing is decreased because of hydrogen desaturation of the whole first wall, which re-absorbs in the successive discharges and partially allows density control. However, the overall trend was recovered at the end of an experiment day indicating that the vacuum conditions are mainly determined by the energy carried into the plasma-wetted areas leading to a depletion of impurities from deeper trapped states. For this reason, only GDC with hydrogen or ion cyclotron resonance heating could speed up the time consuming ECRH wall conditioning. All details of the analysis can be found in [9].

Nevertheless, desaturation of the walls after massive hydrogen operation was successfully demonstrated by means of an intermediate helium ECRH discharge being

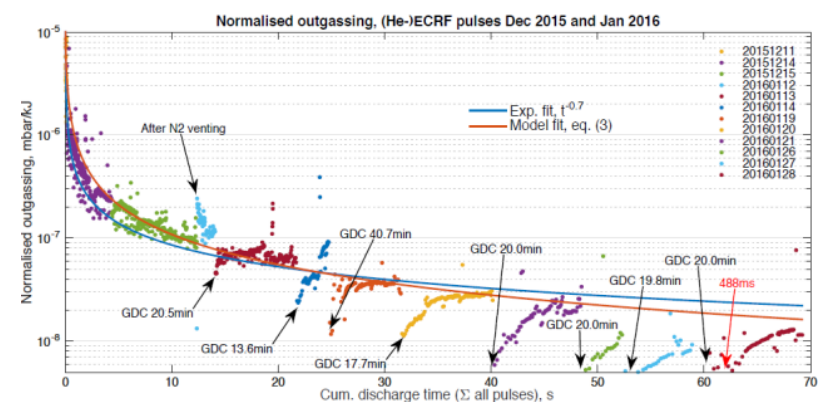

Fig. 7. Outgassing after each ECRH discharge during the first 12 operation days. After this period the fueling gas was changed from $\mathrm{He}$ to $\mathrm{H}_{2}$ with continuation of the trend (as published in [9] and [12])

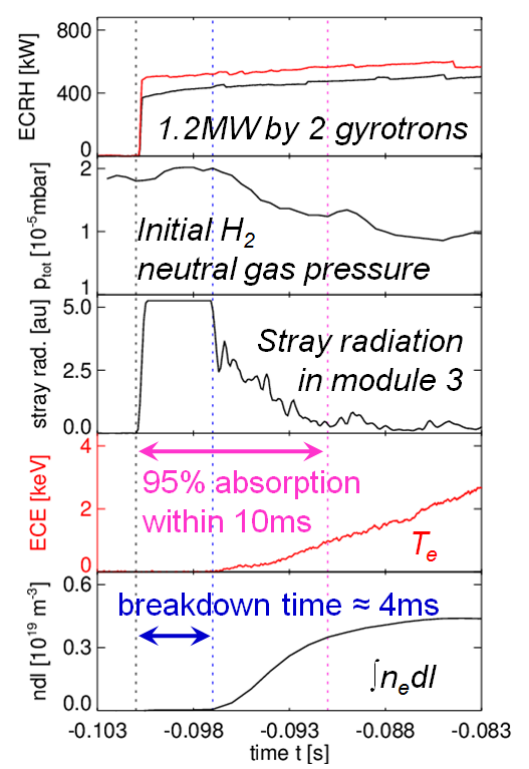

Fig. 8. Temporal evolution of the basic control and plasma parameters during a typical plasma startup in hydrogen

the only possibility to recover density control during the experiment day.

\subsubsection{Startup behaviour \& bulk plasma heating}

The breakdown of W7-X-plasmas happens on a considerably fast time scale. Fig. 8 shows the temporal evolution for a typical startup scenario using 2 gyrotrons with an overall power of $1.2 \mathrm{MW}$. Nearly full absorption can be achieved within $10 \mathrm{~ms}$ after injecting microwaves including a breakdown time of $4 \mathrm{~ms}$. A minimum breakdown time of $2 \mathrm{~ms}$ can be achieved at about $2 \mathrm{MW}$ as can be seen in Fig. 9. In general, the shown breakdown scenario was $100 \%$ reliable, if the used gyrotrons and the gasinlet were activated as programmed.

The ECR heated W7-X plasmas are governed in general by central electron root confinement leading to peaked electron temperature profiles of several $\mathrm{keV}$ and low ion temperatures of about $1 \mathrm{keV}$ at the beginning of a discharge. Further gyrotrons were added after $30 \mathrm{~ms}$ including further gas puffs to increase the density as shown in Fig. 10. Exhausting the energy limit of $4 \mathrm{MJ}$ at 4.3 MW allowed to increase the density up to $\mathrm{n}_{\mathrm{e}}=4 \cdot 10^{19} \mathrm{~m}^{-3}$ at central $\mathrm{T}_{\mathrm{e}}=7 \mathrm{keV}$. Because of the improved heat transfer from electrons to ions with increasing density, the ion temperature rose up to $\mathrm{T}_{\mathrm{i}} \approx 2 \mathrm{keV}$.

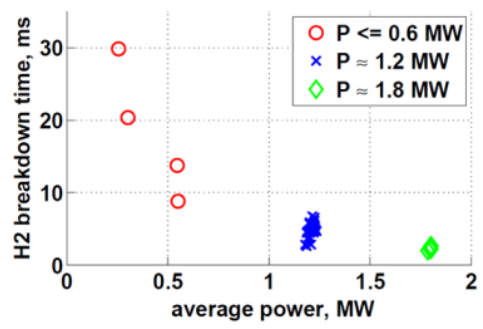

Fig. 9. Breakdown time in $\mathrm{H} 2$-plasmas in dependence on the used ECRH power 


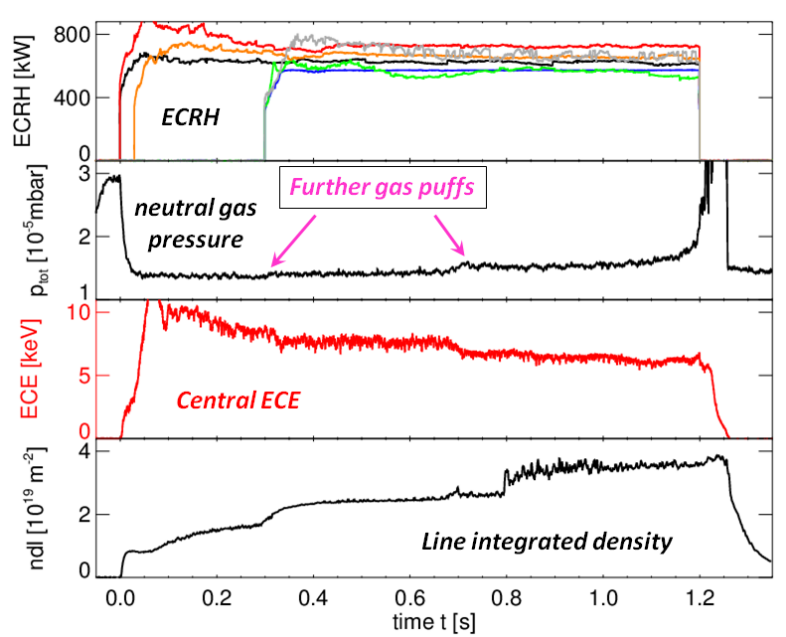

Fig. 10. Temporal evolution of a discharge with the maximum ECRH power of 4.3 MW (after the second gas puff the spike in the density time trace indicates an higher density between $0.7 \mathrm{~s}$ and $0.8 \mathrm{~s}$ due to an error in the interferometer measurement)

\subsubsection{Control of electron temperature \& iota profile}

The spatially localized ECRH power deposition $(\Delta \mathrm{r} / \mathrm{a}<0.1)$ with high power density typically allows the control of non-stiff plasma profiles. For this purpose, the front steering mirrors of the ECRH-beam lines can be varied in the vertical direction. In this case the beams have to be corrected in the horizontal direction to sustain the vertical incidence on the magnetic field lines because of the 3D-plasma shape of W7-X.

Fig. 11 shows a conducted scenario, where the plasma was ignited by 2 on-axis gyrotrons followed by a switch to 3 off-axis gyrotrons with a deposition radius of $\mathrm{r} / \mathrm{a}=0.4$. Additionally, the power of each gyrotron was square wave modulated with a frequency of $17 \mathrm{~Hz}$ to analyze the heat transport and compare it with a similar on axis discharge. A first analysis can be found in [11] casting an electron heat diffusivity of expected magnitude, albeit with error bars too high for an detailed analysis. However, a closer look onto the Thomson profiles (see Fig. 12) of an on- and off-axis discharge with a power level of $0.6 \mathrm{MW}$ shows non-stiff temperature profiles and an increase of the central

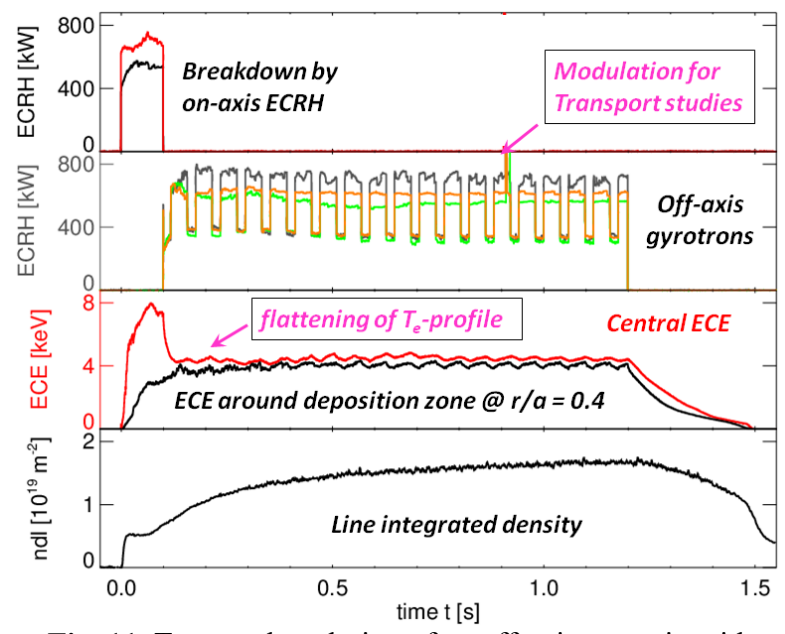

Fig. 11. Temporal evolution of an off-axis scenario with modulated gyrotrons @1.9 MW for transport studies
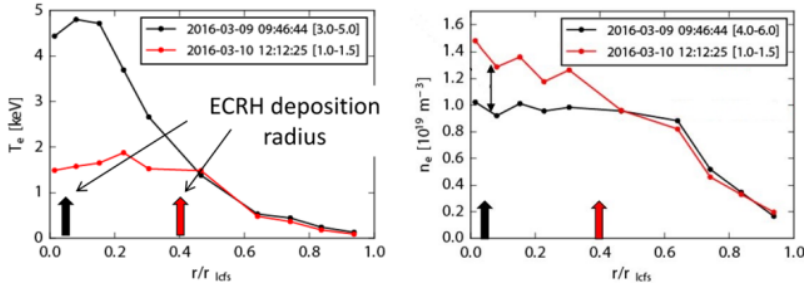

Fig. 12. $T_{e}$ and $n_{e}$ profiles of an on- and off-axis heated discharge@0.6 MW heating power (as published in [12])

density by about $30 \%$ going from on-axis to off-axis. This could indicate a reduction of an outward particle diffusion, which is predicted by neoclassical theory in presence of a strong electron temperature gradient [10]. A more detailed investigation on this topic can be conducted with the availability of more advanced diagnostics in the next operation phase (OP1.2) allowing a more accurate determination of the central radial electric field, which plays a fundamental role in the present long mean-free-path regime. A summary of the actual state of knowledge can be found in [12].

A much more drastic change of the central confinement properties can be triggered by varying the rotational transform by ECCD. Fig. 13 shows the comparison of 2 discharges with a centrally driven current co- and counter-acting to the rotational transform with a central value of $\mathfrak{t}=0.79$ for the vacuum configuration. The central electron temperature crashes in both cases on a timescale of $\mu$ s similar to sawtooth crashes in tokamaks. The reason could be an instability on a rational flux surface, which forms a reconnection of magnetic field lines, because of the central iota changing to the resonant value of 1.0 (co-ECCD) and 0.5 (counterECCD), respectively. The resulting flattening of the central profile would also short-wire the induced central current peaks, so that the confinement can be recovered leading to a rise of the profiles until the next crash. These fast internal current changes are not visible by the rogowski coils, because of slowly varying shielding currents with a L/R-time of several seconds [3].

A possible application in later operation phases could be a controlled reduction of a central impurity contamination, because the typically flat density profile would not be affected by a central profile short circuit. A further possibility would be the flattening of hollow density profiles which are predicted for high density operation [10].

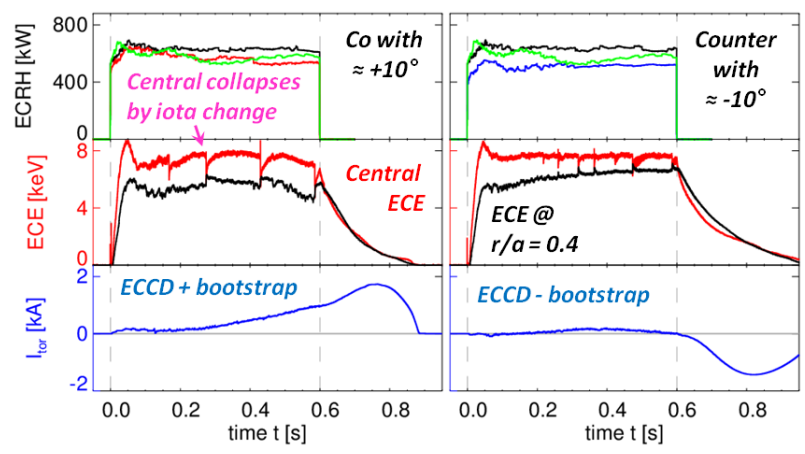

Fig. 13. Comparison of two ECCD discharges@1.8 MW with $\mathrm{Co}$ - and Counter-driven currents to the rotational transform (as published in [12]) 


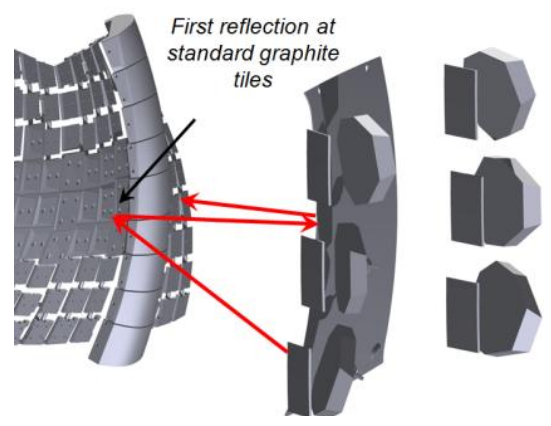

Fig. 14. 3-beampath-scenario used in OP1.1 to increase the ECRH-absorption in O2-heating scenarios

\subsection{O2-heating}

Even though the achieved electron densities in OP1.1 were far away from the cut-off for X2-heating, an advanced O2-heating scenario, foreseen for later operation phases, was proven. In contrast to X2-heating, full absorption of the $\mathrm{O} 2$-mode is not possible for the expected plasma parameters of $\mathrm{n}_{\mathrm{e}} \approx 10^{20} \mathrm{~m}^{-3}$ and $\mathrm{T}_{\mathrm{e}}<5 \mathrm{keV}$ at a heating power of up to $10 \mathrm{MW}$. The shinethrough power is of the order $30 \%$ depending on the particular beam path which is with an incidence angle of about $78^{\circ}$ already optimized to maximum. For this reason, the shinethrough beam should be reflected at an HFS-tile, redirecting the beam again through the plasma center onto a polished stainless steel panel in between two neighbouring ECRH-launchers (see Fig. 14). The second reflection on the panel should pass the plasma again centrally, so that the non-absorbed power will be less than $5 \%$.

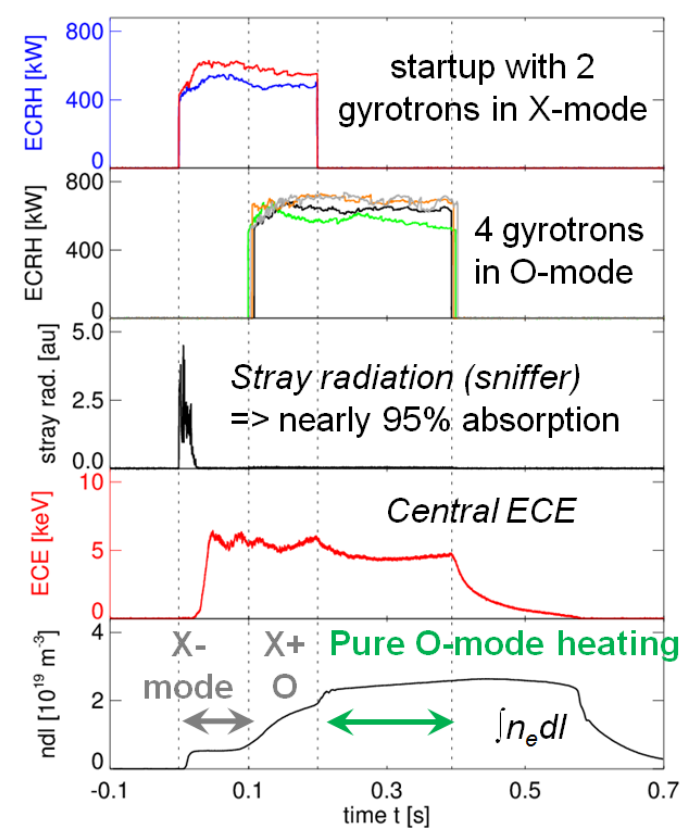

Fig. 15. Temporal evolution of a discharge in which the plasma was sustained by pure O-mode heating @, 2.7 MW using a 3-path-scenario (as published in [12])
The experiment (shown in Fig. 15) was conducted at higher magnetic field of $\mathrm{B}_{00}=2.57 \mathrm{~T}$ to centralize the broad $\mathrm{O} 2$ deposition zone. The plasma breakdown was lower axis magnetic field. After a further gas puff the $\mathrm{O}$ mode gyrotrons were activated at $100 \mathrm{~ms}$ leading to a clear increase of the density. The $\mathrm{X}$-mode gyrotrons were deactivated at $200 \mathrm{~ms}$ with a slight drop in the electron temperature. However, the density increases further and the plasma can be fully sustained by $\mathrm{O} 2$ heating.

\section{Conclusions and outlook to OP1.2}

Even though the plasmas of OP1.1 were typically influenced by many impurities due to the restricted use of glow discharge cleaning and the limited input energy allowed per pulse, the feasibility of the basic ECRH scenarios important for OP1.2 could be proven. However, the O2-heating scenario in OP1.2 will make use of holographic tungsten reflection tiles at the HFS, as already demonstrated at ASDEX Upgrade [13], to provide all 10 gyrotrons with an efficient triple-pass path through the plasma center. In addition, two so-called remote steering launchers have been installed in a toroidal position where the magnetic field gradient is low. These enable advanced current drive scenarios with beneficial ECRH coupling to suprathermal passing electrons [14].

This work has been carried out within the framework of the EUROfusion Consortium and has received funding from the Euratom research and training programme 2014-2018 under grant agreement No 633053. The views and opinions expressed herein do not necessarily reflect those of the European Commission.

\section{References}

1. G. Grieger, et al., Phys. Fluids B 42081 (1992)

2. V. Erckmann, et al., Fusion Sci. Technol. 52291 (2007)

3. T. Sunn Pedersen, et al., Nucl. Fusion 55126001 (2015)

4. G. Gantenbein, et al., J Infrared Milli Terahz Waves 32 320 (2011)

5. D. Moseev, et al., Rev. Sci. Instr. 87083505 (2016)

6. S. Schmuck, et al., Fusion Eng. Des. 841739 (2009)

7. R. König, et al., J. Instrum. 10 P10002 (2015)

8. N. B. Marushchenko, et al., Comp. Phys. Commun. 185 165 (2014)

9. T. Wauters, et al., 43rd EPS Conference on Plasma Physics, Leuven, Belgium, P4.047 (2016)

10. H. Maasberg, et al., Plasma Phys. Control. Fusion 411135 (1999)

11. U. Höfel, et al., 43rd EPS Conference on Plasma Physics, Leuven, Belgium, P4.008 (2016)

12. R. Wolf, et al., Nucl. Fusion 57 102020, 2017

13. D. Wagner, et al, J Infrared Milli Terahz Waves 37:45-54 (2016)

14. N. B. Marushchenko, et al., AIP Conference Proceedings 1580518 (2014) 